\title{
Vehicle Parking Security System with Face Recognition Detection Based on Eigenface Algorithm
}

\author{
Nia Maharani Raharja ${ }^{1 *}$, Muhammad Arief Fathansyah ${ }^{2}$, Anna Nur Nazilah Chamim ${ }^{3}$ \\ ${ }^{1}$ Department of Informatic Engineering, UIN Sunan Kalijaga Yogyakarta, Yogyakarta, Indonesia \\ 2, 3 Department of Electrical Engineering, Universitas Muhammadiyah Yogyakarta, Yogyakarta, Indonesia \\ Email: ${ }^{1}$ nia.raharja@uin-suka.ac.id, ${ }^{2}$ fathanarief@gmail.com, ${ }^{3}$ anna_nnc@yahoo.co.id \\ *Corresponding Author
}

\begin{abstract}
RFID (Radio Frequency Identification) card technology is used for intelligent parking systems. Officers no longer need to record and check manually and repeatedly every vehicle that comes in and out with this system. The system has a weakness, namely if the RFId card is dropped or lost, the person who finds it can use the card. For the purpose of increasing security for users, one of the latest technologies is the use of facial recognition methods. The eigenface algorithm system is an algorithm used for face recognition. The algorithm is used as a training process for the previously inputted employee faces. The results of the training data are stored in a database which is then used as a key to recognize the face of the owner of the motorized vehicle who took the motorcycle. In the trial results of the data application, motorized vehicle owners enter when the facial recognition level is above $70 \%$.
\end{abstract}

Keywords-RFID; Parking Security; Face Recognition; Face Detection; Eigenface Algorithm

\section{INTRODUCTION}

A good parking system will support public facilities used by many parties so that the expectations of all stakeholders can be fulfilled, namely security, convenience and comfort. Several previous researchers have conducted research on intelligent parking systems. Genetic Algorithm Approach for Autonomous Intelligent Vehicle Parking System researched by Thomas [1]. Smart occupancy detection for street traffic parking uses extreme machine learning which was deep researched by Yamin Siddiqui [2]. Improving the prediction of parking availability in smart cities with IoT and ensemblebased models researched by Koumetio Tekouabou [3]. Characteristics of On-street Parking On-street Parking on Urban Streets in Al-Najaf City was investigated by [4]. Model-Based Runtime Monitoring of a Smart City System was investigated by Errousso [5]. DSRC Based Sensor Coalition Protocol for Connected Vehicles in Future Smart Cities researched by El-Said [6]. Review of Smart Parking Using the Internet of Things (IoT) was researched by Rupani [7]. Let My Car Alone: Parking Strategy with Social Distance in the COVID-19 Era researched by Delot [8]. Real Time IP Camera Parking Occupancy Detection using Deep Learning was researched by Farley [9]. Systematic Literature Review of Smart Cities Information Services to Support the Mobility of Persons with Disabilities researched by Rocha [10]

Parking Information Guidance System and Application of Intelligent Technology Used in Urban Areas and Multi-storey
Parking Lots were researched by Hanzl [11]. Parking behavior cluster analysis: A case study in Munich was investigated by Arjona [12]. Designing an integrated smart parking application was researched by Fabusuyi [13]. Smart parking system: a comprehensive review based on various aspects researched by Fahim [14]. Challenges and dilemmas in strategic urban experiments. The analysis of four living laboratories of cycling innovations was investigated by Waes [15]. Feasibility assessment of an emerging smart urban paratransit solution: The case of a taxi aggregator in the city of Kolkata, India investigated by Basu [16]. Visible light communication for intelligent transportation systems: An overview of the latest technologies researched by Shaaban [17]. On-street parking availability data in San Francisco, from stationary sensors and high-mileage vehicle probes were studied by Bock [18]. Incentive Parking Design in Regions - Conception, Technology and Pricing Policy researched by Hanzl [19] Cluster analysis of parking behavior: A case study in Munich researched by Gomari [20].

One of the parking security facilities that has been implemented is using RFID (Radio Frequency Identification). In parking systems that use RFID technology, officers no longer need to record and check manually and repeatedly every vehicle that comes in and out, because it has been coded where the recording activity, data comparison is done automatically by a computer. Several previous researchers have researched using RFID. e-RFIDuino: An Arduino-based RFID environmental station for monitoring cellular tags was investigated by Cassel [21]. A review of the challenges and barriers to the application of RFID technology in the Health sector was investigated by Abugabah [22]. Evaluation of RFID data-based performance in a production system was investigated by Zhong [23]. The use of RFID tags to monitor resin flow and investigate its effect on the mechanical properties of composites was investigated by Hardi [24]. An RFID-based location-based service framework to warn of black spots for accident prevention was investigated by Wilson [25]. Ruthenium (Ru) doping of zinc oxide nanostructure based on radio frequency identification (RFID) gas sensor for NH3 detection was investigated by Ali [26]. Supporting Time Interval Complex Event Processing in RFID Systems was researched by Zhu [27]. The Application and Adoption of RFID in Health Care for a review on Patient Safety was investigated by Haddara [28]. Cybersecurity in 
the Brain-Computer Interface: The theoretical framework of RFID-based design was researched by Ajrawi [29]. Monitoring the vacuum infusion process with an integrated RFID transponder was investigated by Hardi [30].

The integration and testing of the RFID-enabled Smart Factory concept in the Learning Factory was investigated by [31]. Monitoring through visits of growing pigs with UHFRFID was investigated by [32]. A comparison of the difficulties of overcoming an RFID electronic access control system and overcoming a tumbler pin lock was investigated by Jakub [33]. The application of RFID for process mapping automation was investigated by Urso [34]. A dataset survey on combining RFID with cellular technology for the safety of efficient construction professionals was researched by Osunsanmi [35]. RFID: A Key Technology for Humanity researched by Duroc [36]. The RFID tag on the operation of the fire station was investigated by Galko [37]. In-situ measurement in the distribution chain of iron ore pellets using active RFID technology was investigated by Bergquist [38]. Internet of Things in food safety: Literature review and bibliometric analysis researched by Bouzembrak [39]. Integrating RFID transponders into glass fiber reinforced composites to monitor the curing process of components was investigated by Veigt [40].

But there is a weakness of the parking security system using RFID, namely if the portable device that is given the RFId is dropped or lost, the person who finds it can use the tool. For the purpose of increasing security for users, one of the latest technologies, namely the use of face recognition, is used for the contribution of this paper. Face recognition is a biometric technique that allows computers or authentic machines to recognize human faces by recognizing, recording, comparing data and making decisions. This tool is used so that the parking system in a place is safer.

\section{FACE RECOGNITION}

Face recognition is a computer technology to determine the location of the face, face size, detection of facial features and ignoring background images, then identification of facial images is carried out. Face recognition involves many variables, such as source images, image processing results, extracted images and profile data of a person. It also takes a sensing device in the form of a camera sensor and a method to determine whether the image captured by the webcam is classified as a human face or not, as well as to determine profile information that matches the intended face image. Based on several previous studies, the researchers raised the topic of facial recognition systems. Face Recognition using Haar Cascade and LBP Classifiers was studied by Shetty [41]. Effective component-based age-invariant face recognition using Discriminant Correlation Analysis was investigated by Boussaad [42]. Local binary patterns based on directional wavelet transforms for pose-invariant facial expression and recognition were investigated by Muqeet [43]. Clustering and Jarque-Bera Normality Tests for Face Recognition were studied by Abdellatif [44]. Transfer learning for facial recognition using fingerprint biometrics was investigated by Kute [45]. Improved facial recognition algorithms and their application in attendance management systems were investigated by Bah [46]. An open source facial recognition framework was investigated by Csaba [47]. Extraction of Feature Density from Areas Important for Face Recognition was investigated by Vinay [48]. A lightweight, end-to-end Pose-Robust facial recognition system with Deep Residual Equivalent Mapping was studied by Gunawan [49]. Deep learning-based descriptors in applications for aging problems in facial recognition were investigated by Boussaad [50]

Deep facial recognition using imperfect facial data was studied by Elmahmudi [51]. Towards the facial recognition of pigs in farms using convolutional neural networks researched by Hansen [52]. An approach to improve facial recognition performance that does not change based on age based on gender classification was investigated by Nayak [53]. Human face recognition with a combination of DWT and machine learning was investigated by Tabassum [54]. Energy Saving Face Recognition in Mobile-Fog Environment was investigated by Mukherjee [55]. Automatic facial recognition of rhesus macaques was studied by Witham [56]. The Survey on Human Facial Expression Recognition Technique was researched by Revina [57]. The long arm of the algorithm? Automatic Face Recognition as evidence and trigger for police intervention was investigated by Kotsoglou [58]. XNORNet and Minimum Barrier Detection for Efficient Face Recognition were investigated by Bharadwaj [59]. Face recognition using Radial Angle Transformation was studied by Hamdan [60].

The design of the face recognition block diagram is shown in Fig. 1. The figure shows that the block diagram of the face recognition method is composed of three parts: face detection, feature extraction and face recognition.

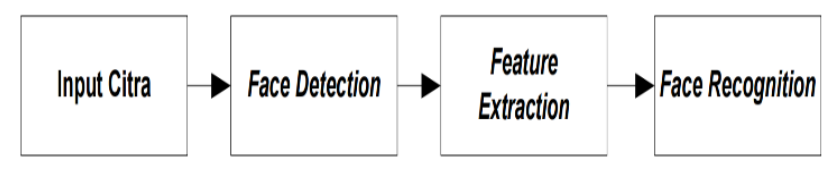

Fig. 1. Face recognition block diagram

\section{A. Face Detection}

Face detection in this study uses a method that is divided into four main components: Haar Like Feature, Integral Image, Adaptive Boosting and Cascade of Classifier.

\section{Haar Like Feature}

Haar-Like Feature is a method used to detect objects shown in Fig. 2. The figure shows that the Haar Like feature scheme, which processes images in a grid area, contains several pixels from the image portion.

The pixels in one area are summed and a calculation process is carried out (reducing the average pixel value in the light and dark parts of the box) so that the unique value difference is obtained in each area of the boxes.

The pixels in one area are added up by the equation (1) as

$$
F_{\text {Haar }}=E\left(R_{\text {white }}\right)-E\left(R_{\text {black }}\right)
$$



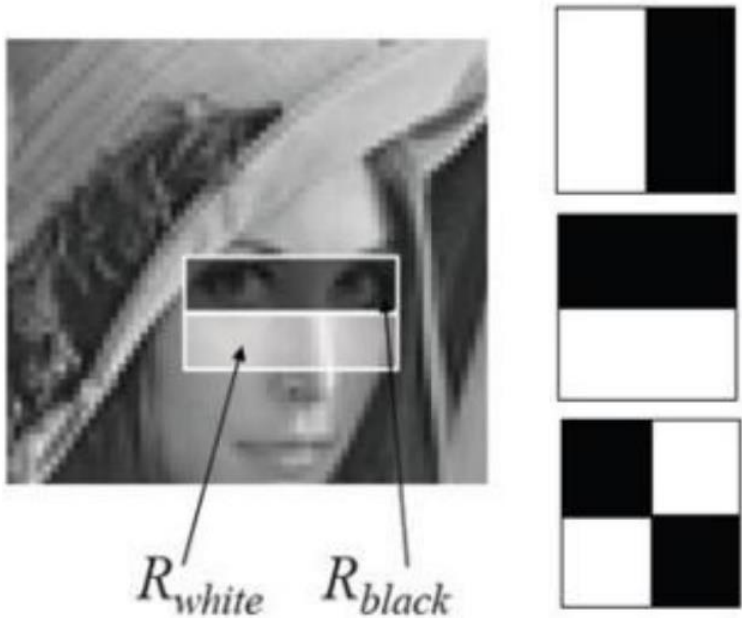

Fig. 2. Schematic of Haar Like Feature

\section{Integral Image}

An integral image is an image whose value for each pixel is the accumulation of the values of the top and left pixels. For example, pixel $\left(x^{\prime}, y^{\prime}\right)$ has an accumulative value for all pixels $(x, y)$ where $x^{\prime} \leq x$ and $y^{\prime} \leq y$.

$$
i i(x, y)=\sum_{x^{\prime} \leq x, y^{\prime} \leq y} i\left(x^{\prime}, y^{\prime}\right)
$$

Where $i i(x, y)$ is the integral image and $i\left(x^{\prime}, y^{\prime}\right)$ is the original image with condition.

\section{Adaptive Boosting}

In general, boosting is adding a weak learner to one strong learner. For each iteration, one weak learner learns from a training data. Then the weak learner is added to the strong learner. After the weak learners are added, the data is then changed for each weight. The data that is misclassified will experience additional weight and data that is classified correctly will experience a reduction in weight, therefore the weak learner in the next iteration will be more focused on the data that was misclassified by the previous weak leaner.

$$
F(x)=\sum_{t=1}^{T} \propto_{t} h_{t}(x)
$$

where $h_{t}(x)$ is the basic classifier, $\propto_{t}$ is the learning level, and $F(x)$ is the final classifier.

\section{Cascade of Classifiers}

Classification combined in a Cascade of Classifier is shown in Fig. 3. The figure shows that the speed of the detection process can be increased by focusing only on areas in the image that have a chance. This is done to determine where the location of the object being searched for in an image. The image shows the first level classification, each sub-image will be classified using one feature. This classification will leave approximately $50 \%$ of the subimages to be classified in the second stage. The number of sub-images that pass the classification will be reduced to around $2 \%$.
The cascade structure is useful for rejecting negative subwindows as much and as quickly as possible at the level possible. While a positive example will trigger the evaluation of each classifier in the cascade. Subsequent classifiers are trained using examples that go through all the previous stages.

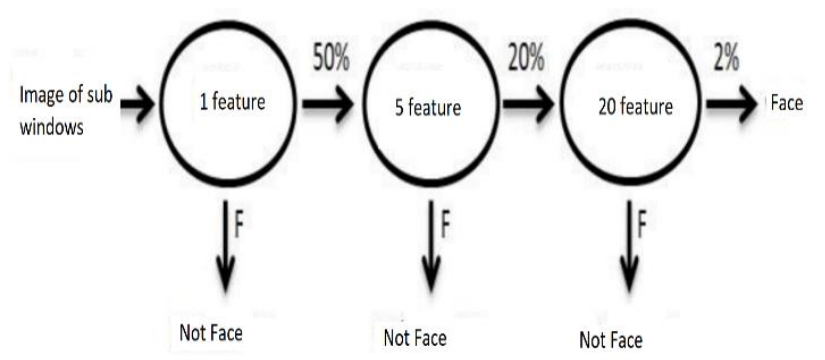

Fig. 3. Cascade of Classifier

\section{B. Face Recognition}

The basic principle of facial recognition is to quote unique face information, then encode and compare it with the decoded results that were previously performed. In the eigenface method, decoding is done by calculating the eigenvector and then representing it in a matrix. Eigen vectors are also expressed as facial characteristics, therefore this method is called eigenface. Each face is represented in a linear combination of eigenfaces. The eigenface method is how to decipher the relevant information from a face image, then convert it into a set of the most efficient codes and compare the face code with a database containing various faces that have been coded similarly.

PCA eigenfaces are used to reduce the dimensions of a set or image space so that the new base or coordinate system can better describe the typical model of the set. The desired model is a set of trained faces. The new features will be formed through a linear combination. The components of this character space feature will not be correlated with each other and will maximize the differences that exist in the original variables.

Broadly speaking, the steps of the PCA method are as follows:

- Find the matrix u

- Find the covariance matrix:

- $\quad$ Find the eigen values (ë) and eigen vector (V) of the matrix $\mathrm{C}$

- Find the eigenface matrix The eigenface matrix can be used for image recognition.

\section{METHOD}

The flow diagram of the facial recognition system application for motorized vehicle parking is shown in Fig. 4. The image shows that the image is obtained from the webcam sensor, the RGB color image is converted into a grayscale image to reduce the color depth. From the grayscale image, face detection is carried out using the Adaboost method, if the image is not detected as a face, then capture the image by the webcam sensor repeatedly. If part of the image is detected as a face by the face detection engine then proceed with the next two options: 
- Perform personal profile data storage into the database as well as save the face image to a file for comparison.

- If previously the face image and profile data have been stored in the database, then do face identification using the Eigeface PCA method.

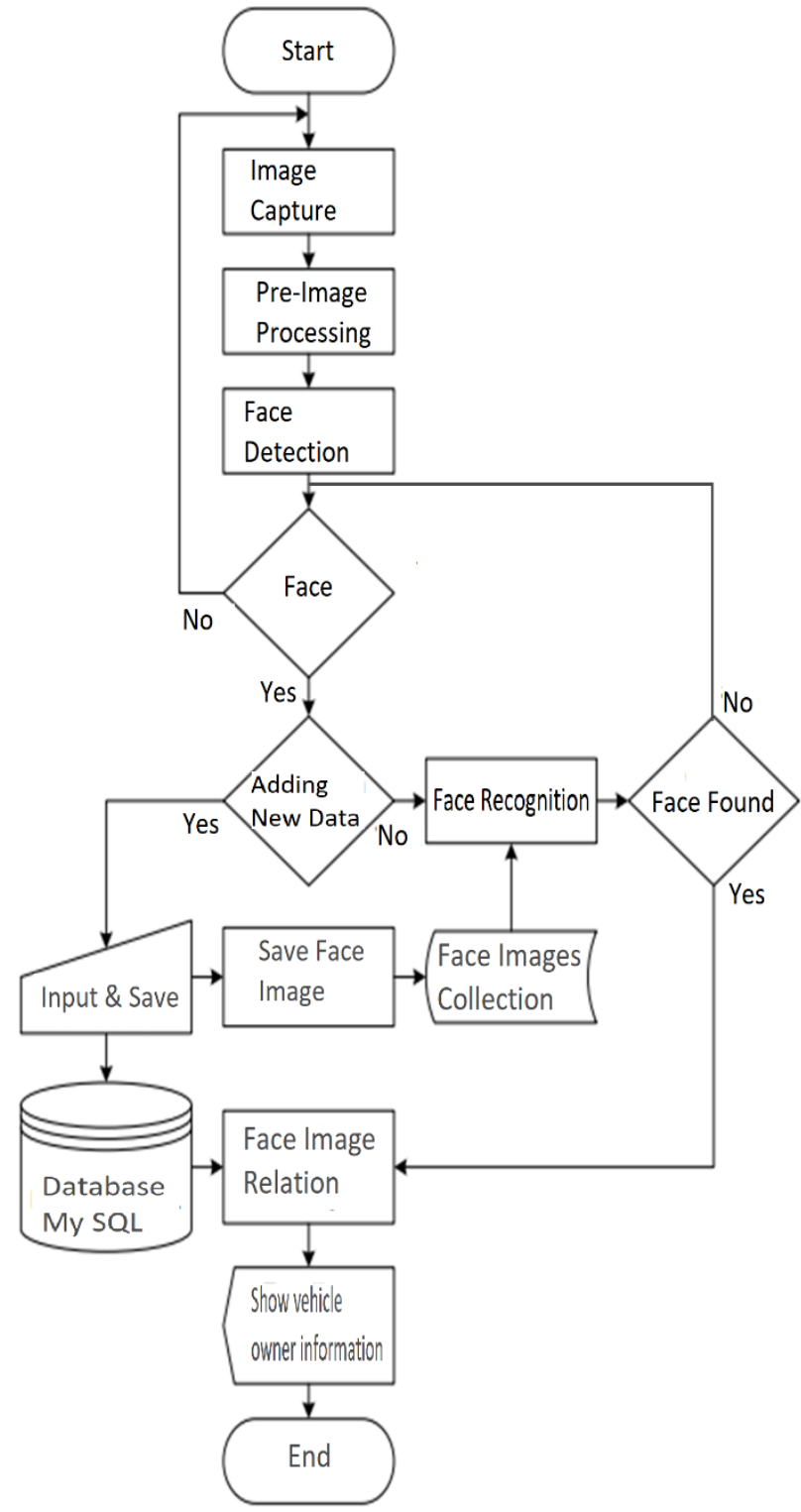

Fig. 4. Facial Recognition Workflow

The face detection flowchart is done by classifying an image, after previously a classifier was formed from the training data shown in Fig. 5. The image shows that the grayscale input is processed using Haar Like Feature with the data set of Feret faces. The output of the haar like feature method is reprocessed using the Integral image, adaptive image, adaptive boosting and Cascade of classifier theodes. Then the image is compared with the data set of Feret faces.

The Face Recognition Algorithm is shown in Fig. 6. There are six steps of the Face Recognition Algorithm which are explained from the Figure. The first step of the algorithm is to get a set of $\mathrm{S}$ with $\mathrm{M}$ face images. For example: $\mathrm{M}=25$. Each image is transformed into a vector of size $\mathrm{N}$ and placed into the set represented by the equation (4) as

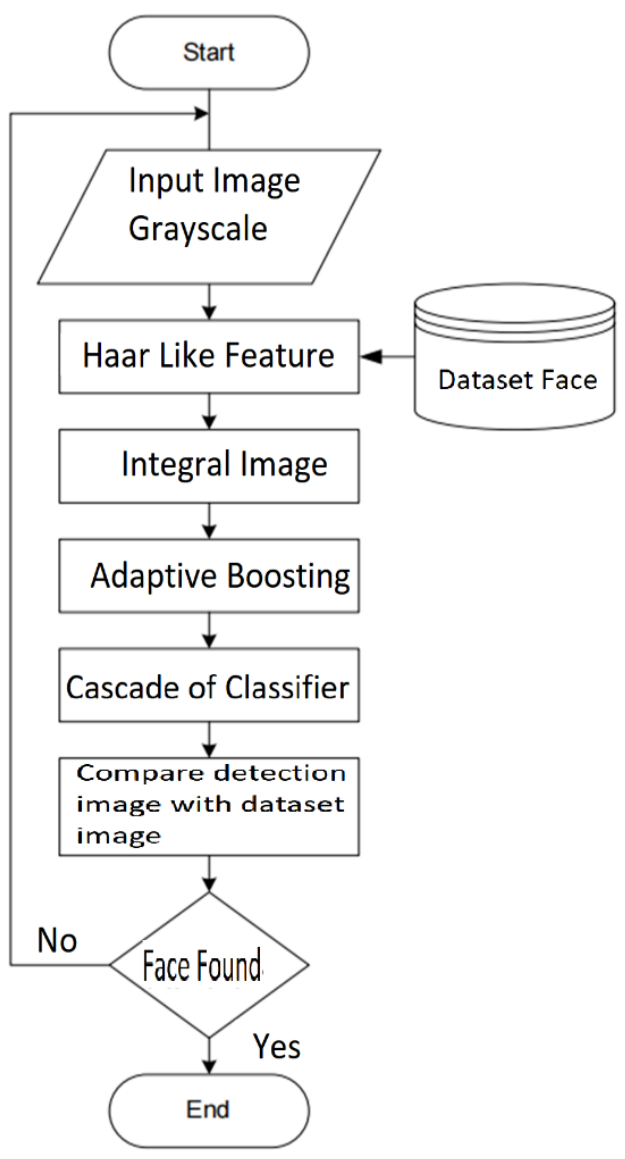

Fig. 5. Face Detection Algorithm

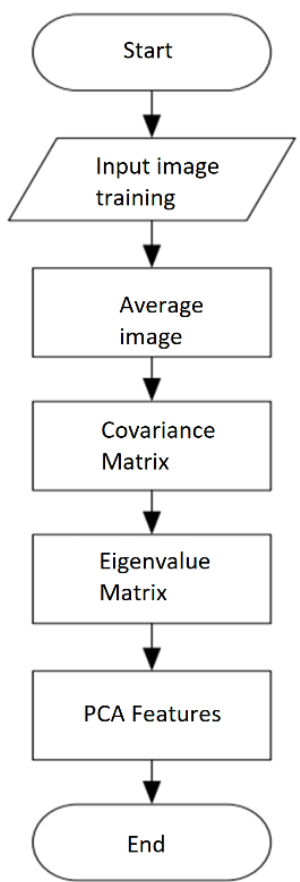

Fig. 6. Face Recognition Algorithm

$$
S=\left\{\Gamma_{1}, \Gamma_{2}, \Gamma_{3}, \cdots, \Gamma_{M}\right\}
$$

After getting the set, it will get the average image (mean image) $\psi$. 


$$
\psi=\frac{1}{M} \sum_{n=1}^{M} \Gamma_{n}
$$

Then the difference $\phi$ between the input image and the average image is obtained.

$$
\phi_{i}=\Gamma_{i}-\psi
$$

Next, look for the set of $M$ orthonormal vectors, un, which best describes the distribution of the data. The Kth vector, uk, is chosen as such.

$$
\lambda_{k}=\frac{1}{M} \sum_{n-1}^{M}\left(u_{k}^{T} \phi_{n}\right)^{2}
$$

Note: $u_{k}$ is the eigenvectors of the covariance matrix $C$ and $\mathrm{k}$ is the eigenvalues of the covariance matrix $C$. Then the covariance matrix $C$ is obtained in the following way

$$
C=\frac{1}{M} \sum_{n=1}^{M} \phi_{n} \phi_{n}^{T}
$$

The facial recognition procedure in Eigenfaces Principal Component Analysis is as follows:

- Converting new faces into eigenface components. First, the input images are compared with the average images and their differences are multiplied by the respective eigenvectors of the $\mathrm{L}$ matrix. Each value will represent a weight and will be stored in the vector $\Omega$.

- Determine which face class gives the best picture for the input image. This is done by minimizing the Euclidean distance.

$$
\varepsilon_{k}=\left\|\Omega-\Omega_{k}\right\|
$$

The input face considers class categorization. If $\varepsilon_{k}$ is below the threshold then the image is a recognized face. If the difference is above the given threshold, but below the second threshold, then the image is an unrecognized face. If the input image is not part of the two thresholds above, then the image is not a face.

\section{RESULTS AND DisCUSSION}

The facial recognition architecture is shown in Fig. 7. The image consists of two main parts, namely the interface and the server. The interface is the initial appearance of the program in the form of access rights authority for users. In the server there are two main parts, namely the model and data source, the model is a face recognition method or algorithm, consisting of face detection and face recognition related to the data source in the form of a FERET face dataset (in the form of an XML file) when face detection is performed, profile data stored in a MySQL database and the face file is a face image in JPEG format.

The program interface functions as a bridge between the user and the system to interact as shown in Fig. 8. The image is a GUI image when users enter their data. The data entered is the name of the owner of the motorized vehicle and the license plate number.

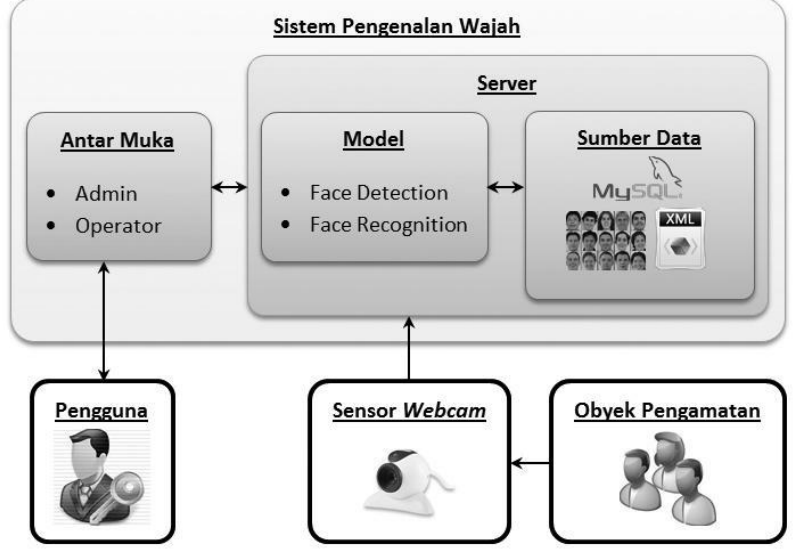

Fig. 7. Facial Recognition System Architecture

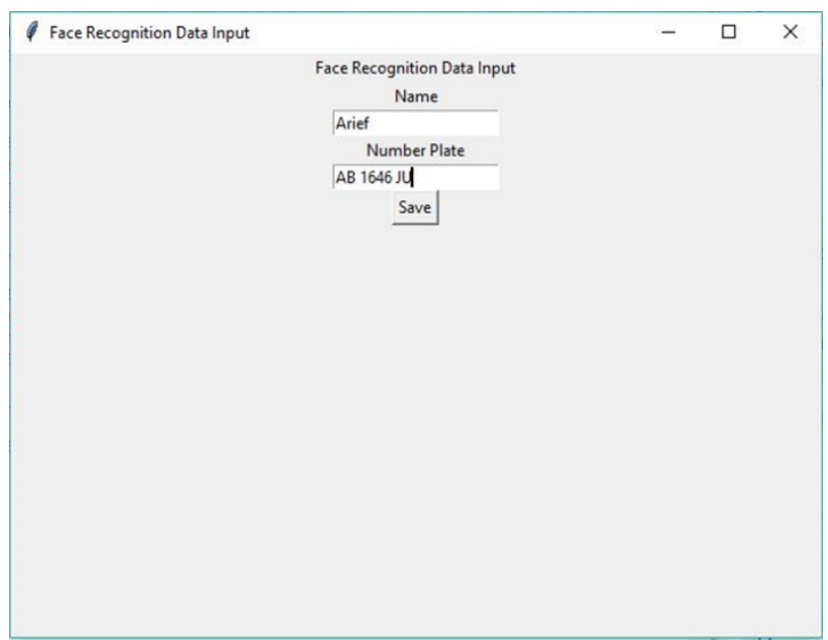

Fig. 8. Face Recognition Data Input

The camera automatically takes pictures of the user's face up to 21 times after the data is entered and the user presses the save button as shown in Fig. 9. The picture shows that there are 21 photos of motorized vehicle owners. After taking the photo, the data is entered into the database. In order to be detected by the program, this program must be trained first, after being trained, it will be able to detect the face of the user.
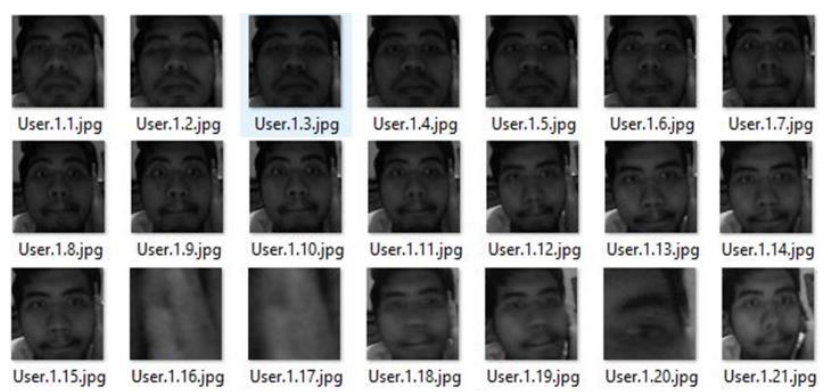

Fig. 9. Taking 21 photos of motorized vehicle owners

The program for security checks used at the exit location is shown in Fig. 10. The figure displays a GUI if the data from the user has been entered into the Database and trained in the Face Recogniton program. Fig. 10 is an example of a GUI display. 


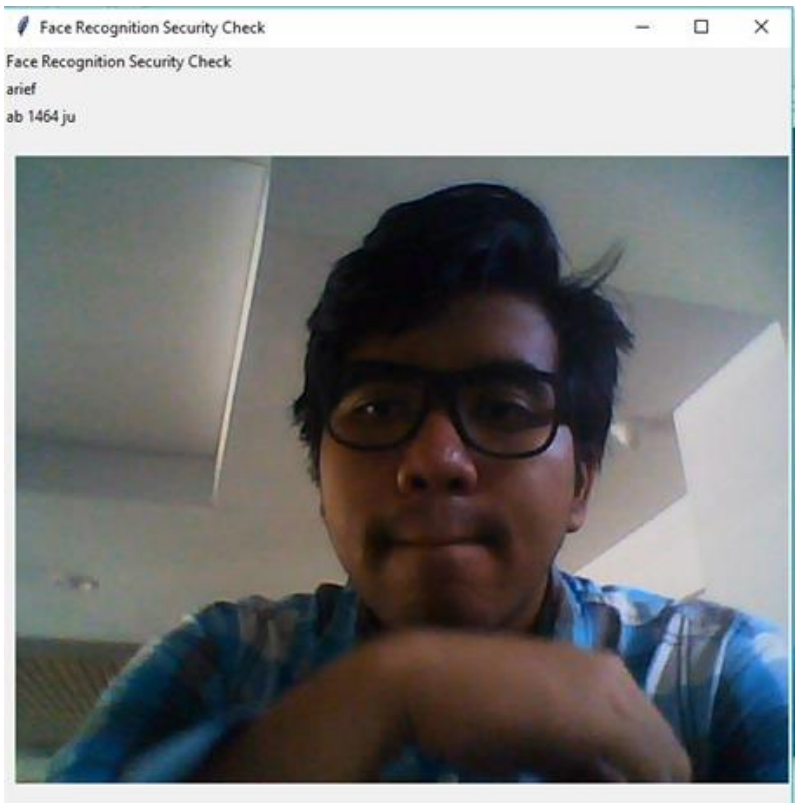

Fig. 10. Face Recognition Security Check

The test was carried out on 10 respondents consisting of 5 men and 5 women shown in Fig. 11. The figure shows that there are five tests, namely facial recognition testing based on facial position, facial recognition testing based on lighting levels, facial recognition testing based on facial expressions, testing facial recognition based on object distance and webcam sensors, and facial recognition testing based on different attributes, for example: mustache, glasses, hairstyle and beard. The facial recognition test based on the position of the face produces a truth rate of $73 \%$ and an error rate of $27 \%$. The face recognition test is based on the lighting level of $90 \%$ truth level and $10 \%$ error rate. The facial recognition test is based on facial expressions with a truth rate of $73 \%$ and an error rate of $27 \%$. Face recognition test based on object distance and webcam sensor correctness rate of $83 \%$ and error rate of $17 \%$. Face recognition testing is based on different attributes, for example: mustache, glasses, hairstyle and beard, $80 \%$ correctness rate and $20 \%$ error rate. Average value of validity $80 \%$ truth rate and $20 \%$ error rate.

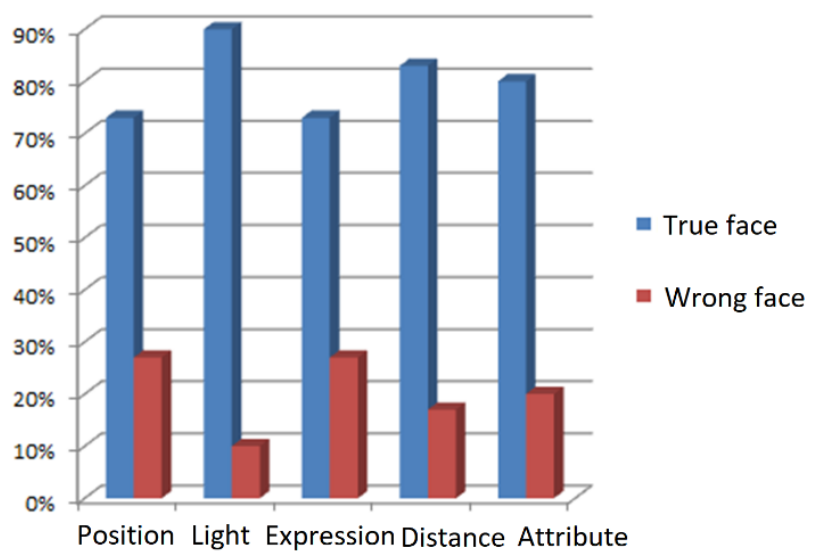

Fig. 11. Face recognition system validity graph.

If the light intensity on the face image object is above 1700 lux then the face image object can be recognized properly, but if the intensity ranges from 0 to 1700 lux then the face image is not recognized at all or is wrong in detecting faces due to lack of lighting.

If the lighting on the face image object is in normal conditions (more than $1700 \mathrm{lux}$ ), then the addition of facial attributes, including: hijab, sideburns, hairstyles and beards, facial image objects can still be recognized properly. Meanwhile, the addition of glasses and mustache attributes can affect the quality decline.

Face recognition in terms of rotational position is very influential on the accuracy of face recognition. There are 3 possible types of facial rotation, including: turning left or right, shaking left or right, and looking up or looking down. Ideally the face that can be identified well is the face facing the camera sensor perpendicularly. The incline that can be tolerated is around 10 degrees from the normal position. But normally (not physically disabled) most faces are perpendicular to the web camera sensor so that the application still detects faces well.

Facial recognition is also influenced by facial expressions, the expressions that are tried are smiling (the corners of the lips are slightly up), laughing (showing teeth), and sad (pouting and the corners of the lips are flat or down). From the mimic test, it turned out to be quite significant in influencing the application in recognizing faces.

Judging from the distance between the face object and the webcam sensor, the application can effectively recognize faces at a distance of 0.30 meters to 2.5 meters when using a standard web camera on a laptop (built-in webcam). Face recognition can be carried out further (approximately 6 meters to 10 meters) depending on the quality of the webcam sensor image resolution and zooming capability when the face object is very far away.

The time required for the computer to identify faces (recognition to identification/labeling of faces) in real time is very fast, which is less than 1 second. As a test, the application uses 10 face images as training data and each object has 5 different facial image positions and the accuracy of face recognition reaches $80 \%$.

\section{CONCLUSION}

The eigenface method contained in the OpenCV library which is an open source library is used in researching parking security systems with face recognition detection. From this research, it can be concluded that the level of lighting and the distance between the camera and the object in taking pictures of faces can affect image quality and training results. In trials conducted with two different distances. The closest distance gives more accurate results than the longer distance. When the distance from the face to the camera is getting closer, the structure of the face shape is getting clearer and gives the characteristics of a particular person's face. The role of lighting also clarify the shape or structure of a person's face. Combining the ideal distance and exposure gives more accurate results. The average success rate of face recognition with the Adaboost and Eigenfaces PCA methods reaches $80 \%$ in different conditions (distance of objects to sensors, lighting, position, attributes, and facial expressions). When compared with other methods, face recognition using the Adaboost and Eigenfaces PCA methods has the advantage of 
speeding up the decision-making process to recognize faces in real time conditions.

\section{REFERENCES}

[1] D. Thomas and B. C. Kovoor, "A Genetic Algorithm Approach to Autonomous Smart Vehicle Parking system," Procedia Comput. Sci., vol. 125, pp. 68-76, 2018.

[2] S. Yamin Siddiqui, M. Adnan Khan, S. Abbas, and F. Khan, "Smart occupancy detection for road traffic parking using deep extreme learning machine," J. King Saud Univ. - Comput. Inf. Sci., no. xxxx, pp. 1-7, Feb. 2020.

[3] S. C. Koumetio Tekouabou, E. A. Abdellaoui Alaoui, W. Cherif, and H. Silkan, "Improving parking availability prediction in smart cities with IoT and ensemble-based model," J. King Saud Univ. - Comput. Inf. Sci., no. xxxx, pp. 1-11, Feb. 2020.

[4] H. A. Eedan Al-Jameel and R. R. Muzhar, "Characteristics of Onstreet Parking On-street Parking in Al-Najaf City Urban Streets," Transp. Res. Procedia, vol. 45, no. 2019, pp. 612-620, 2020.

[5] H. Errousso, N. Malhene, S. Benhadou, and H. Medromi, "Predicting car park availability for a better delivery bay management," Procedia Comput. Sci., vol. 170, pp. 203-210, 2020.

[6] M. El-Said, S. Mansour, and V. Bhuse, "DSRC Based SensorPooling Protocol for Connected Vehicles in Future Smart Cities," Procedia Comput. Sci., vol. 140, pp. 70-78, 2018

[7] S. Rupani and N. Doshi, "A Review of Smart Parking Using Internet of Things (IoT)," Procedia Comput. Sci., vol. 160, pp. 706-711, 2019.

[8] T. Delot and S. Ilarri, "Let My Car Alone: Parking Strategies with Social-Distance Preservation in the Age of COVID-19," Procedia Comput. Sci., vol. 177, pp. 143-150, 2020.

[9] A. Farley, H. Ham, and Hendra, "Real Time IP Camera Parking Occupancy Detection using Deep Learning," Procedia Comput. Sci., vol. 179, no. 2020, pp. 606-614, 2021.

[10] N. P. Rocha, G. Santinha, A. Dias, C. Rodrigues, M. Rodrigues, and A. Queirós, "A Systematic Literature Review of Smart Cities' Information Services to Support the Mobility of Impaired People," Procedia Comput. Sci., vol. 181, no. 2019, pp. 182-188, 2021

[11] J. Hanzl, "Parking Information Guidance Systems and Smart Technologies Application Used in Urban Areas and Multi-storey Car Parks," Transp. Res. Procedia, vol. 44, no. 2019, pp. 361-368, 2020.

[12] J. Arjona, M. Linares, J. Casanovas-Garcia, and J. J. Vázquez, "Improving Parking Availability Information Using Deep Learning Techniques,” Transp. Res. Procedia, vol. 47, no. 2019, pp. 385-392, 2020.

[13] T. Fabusuyi and V. Hill, "Designing an integrated smart parking application,” Transp. Res. Procedia, vol. 48, no. 2019, pp. 10601071, 2020.

[14] A. Fahim, M. Hasan, and M. A. Chowdhury, "Smart parking systems: comprehensive review based on various aspects," Heliyon, vol. 7, no. 5, p. e07050, May 2021.

[15] A. van Waes, A. Nikolaeva, and R. Raven, "Challenges and dilemmas in strategic urban experimentation An analysis of four cycling innovation living labs," Technol. Forecast. Soc. Change, vol. 172, no. May, p. 121004, Nov. 2021.

[16] A. Basu, "Viability assessment of emerging smart urban para-transit solutions: Case of cab aggregators in Kolkata city, India," J. Urban Manag., vol. 8, no. 3, pp. 364-376, Dec. 2019.

[17] K. Shaaban, M. H. M. Shamim, and K. Abdur-Rouf, "Visible light communication for intelligent transportation systems: A review of the latest technologies," J. Traffic Transp. Eng. (English Ed., vol. 8, no. 4, pp. 483-492, Aug. 2021

[18] F. Bock and S. Di Martino, "On-street parking availaibilty data in San Francisco, from stationary sensors and high-mileage probe vehicles," Data Br., vol. 25, p. 104039, Aug. 2019.

[19] J. Hanzl, "Design of Incentive Parking Lots in the Region Conception, Technology and Pricing Policy," Transp. Res. Procedia, vol. 53, no. 2020, pp. 244-251, 2021.
[20] S. Gomari, C. Knoth, and C. Antoniou, "Cluster analysis of parking behaviour: A case study in Munich," Transp. Res. Procedia, vol. 52, no. 2020, pp. 485-492, 2021.

[21] M. Cassel, O. Navratil, F. Perret, and H. Piégay, "The e-RFIDuino: An Arduino-based RFID environmental station to monitor mobile tags," HardwareX, vol. 10, p. e00210, Oct. 2021.

[22] A. Abugabah, N. Nizamuddin, and A. Abuqabbeh, "A review of challenges and barriers implementing RFID technology in the Healthcare sector," Procedia Comput. Sci., vol. 170, pp. 1003-1010, 2020.

[23] R. Y. Zhong, "RFID Data Driven Performance Evaluation in Production Systems," Procedia CIRP, vol. 81, pp. 24-27, 2019.

[24] E. Hardi, M. Veigt, M. Koerdt, A. S. Herrmann, and M. Freitag, "Use of RFID tags for monitoring resin flow and investigation of their influence on the mechanical properties of the composite," Procedia Manuf., vol. 24, pp. 305-310, 2018.

[25] K. Wilson Cheruiyot, G. Okeyo, and W. O. Ochieng, "RFID-based location based services framework for alerting on black spots for accident prevention," Egypt. Informatics J., no. xxxx, Jun. 2021.

[26] I. Ali, A. E.-H. B. Kashyout, M. Tayel, H. Shokry Hassan, and M. Rizk, "Ruthenium $(\mathrm{Ru})$ doped zinc oxide nanostructure-based radio frequency identification (RFID) gas sensors for NH3 detection," J. Mater. Res. Technol., vol. 9, no. 6, pp. 15693-15704, Nov. 2020.

[27] W. Zhu and S. Sun, "Supporting Interval Time Complex Events Processing in an RFID System," Procedia Comput. Sci., vol. 147, pp. 324-330, 2019.

[28] M. Haddara and A. Staaby, "RFID Applications and Adoptions in Healthcare: A Review on Patient Safety," Procedia Comput. Sci., vol. 138, pp. 80-88, 2018.

[29] S. Ajrawi, R. Rao, and M. Sarkar, "Cybersecurity in BrainComputer Interfaces: RFID-based design-theoretical framework," Informatics Med. Unlocked, vol. 22, no. November 2020, p. 100489, 2021.

[30] E. Hardi, M. Veigt, M. Koerdt, A. S. Herrmann, and M. Freitag, "Monitoring of the vacuum infusion process by integrated RFID transponder," Procedia Manuf., vol. 52, no. 2019, pp. 20-25, 2020.

[31] M. Mladineo, I. Veza, N. Gjeldum, M. Crnjac, A. Aljinovic, and A. Basic, "Integration and testing of the RFID-enabled Smart Factory concept within the Learning Factory," Procedia Manuf., vol. 31, pp. 384-389, 2019

[32] F. Adrion et al., "Monitoring trough visits of growing-finishing pigs with UHF-RFID," Comput. Electron. Agric., vol. 144, no. November 2017, pp. 144-153, Jan. 2018.

[33] D. Jakub and L. Filip, "Comparison of the difficulty overcoming of RFID electronic access control systems and overcoming of pin tumbler locks," Transp. Res. Procedia, vol. 55, pp. 1620-1626, 2021.

[34] O. Urso, F. Chiacchio, L. Compagno, and D. D'Urso, “An RFID application for the process mapping automation," Procedia Manuf., vol. 42, no. 2019, pp. 8-15, 2020.

[35] T. O. Osunsanmi, A. E. Oke, and C. O. Aigbavboa, "Survey dataset on fusing RFID with mobile technology for efficient safety of construction professionals," Data Br., vol. 25, p. 104290, Aug. 2019.

[36] Y. Duroc and S. Tedjini, "RFID: A key technology for Humanity," Comptes Rendus Phys., vol. 19, no. 1-2, pp. 64-71, Jan. 2018.

[37] I. Galko, R. Kuffa, P. Magdolenová, J. Svetlík, and A. Velas, "RFID tags at the operation of fire stations," Transp. Res. Procedia, vol. 55, no. 2019, pp. 941-948, 2021.

[38] B. Bergquist and E. Vanhatalo, "In-situ measurement in the iron ore pellet distribution chain using active RFID technology," Powder Technol., vol. 361, pp. 791-802, Feb. 2020.

[39] Y. Bouzembrak, M. Klüche, A. Gavai, and H. J. P. Marvin, "Internet of Things in food safety: Literature review and a bibliometric analysis," Trends Food Sci. Technol., vol. 94, no. April, pp. 54-64, Dec. 2019.

[40] M. Veigt, E. Hardi, M. Koerdt, A. S. Herrmann, and M. Freitag, "Curing Transponder - Integrating RFID transponder into glass fiber-reinforced composites to monitor the curing of the component," Procedia Manuf., vol. 24, pp. 94-99, 2018. 
[41] A. B. Shetty, Bhoomika, Deeksha, J. Rebeiro, and Ramyashree, "Facial Recognition using Haar Cascade and LBP Classifiers," Glob. Transitions Proc., pp. 0-12, Aug. 2021.

[42] L. Boussaad and A. Boucetta, "An effective component-based ageinvariant face recognition using Discriminant Correlation Analysis," J. King Saud Univ. - Comput. Inf. Sci., no. xxxx, Aug. 2020.

[43] M. A. Muqeet and R. S. Holambe, "Local binary patterns based on directional wavelet transform for expression and pose-invariant face recognition," Appl. Comput. Informatics, vol. 15, no. 2, pp. 163 171, Jul. 2019.

[44] D. Abdellatif, K. El Moutaouakil, and K. Satori, "Clustering and Jarque-Bera Normality Test to Face Recognition," Procedia Comput. Sci., vol. 127, pp. 246-255, 2018.

[45] R. Kute, V. Vyas, and A. Anuse, "Transfer learning for face recognition using fingerprint biometrics,” J. King Saud Univ. - Eng. Sci., no. xxxx, Aug. 2021.

[46] S. M. Bah and F. Ming, "An improved face recognition algorithm and its application in attendance management system," Array, vol. 5, no. December 2019, p. 100014, Mar. 2020.

[47] B. Csaba, H. Tamás, A. Horváth, A. Oláh, and I. Z. Reguly, "PPCU Sam: Open-source face recognition framework," Procedia Comput. Sci., vol. 159, pp. 1947-1956, 2019.

[48] A. Vinay, A. Gupta, A. Bharadwaj, A. Srinivasan, K. N. B. Murthy, and S. Natarajan, "Dense Extraction of Features from Salient Regions for Face Recognition,” Procedia Comput. Sci., vol. 143, pp. 510-518, 2018.

[49] K. W. Gunawan, N. Halimawan, and Suharjito, "Lightweight End to end Pose-Robust face recognition system with Deep Residual Equivariant Mapping," Procedia Comput. Sci., vol. 179, no. 2020, pp. 648-655, 2021.

[50] L. Boussaad and A. Boucetta, "Deep-learning based descriptors in application to aging problem in face recognition," J. King Saud Univ. - Comput. Inf. Sci., no. xxxx, Oct. 2020.
[51] A. Elmahmudi and H. Ugail, "Deep face recognition using imperfect facial data," Futur. Gener. Comput. Syst., vol. 99, pp. 213-225, Oct. 2019.

[52] M. F. Hansen et al., "Towards on-farm pig face recognition using convolutional neural networks," Comput. Ind., vol. 98, pp. 145-152, Jun. 2018.

[53] J. S. Nayak and M. Indiramma, "An approach to enhance age invariant face recognition performance based on gender classification,” J. King Saud Univ. - Comput. Inf. Sci., no. xxxx, Jan. 2021.

[54] F. Tabassum, M. Imdadul Islam, R. Tasin Khan, and M. R. Amin, "Human face recognition with combination of DWT and machine learning," J. King Saud Univ. - Comput. Inf. Sci., no. xxxx, Feb. 2020.

[55] D. Mukherjee, R. Das, S. Majumdar, S. Ghosh, S. Thepade, and A. Basu, "Energy Efficient Face Recognition in Mobile-Fog Environment," Procedia Comput. Sci., vol. 152, pp. 274-281, 2019.

[56] C. L. Witham, “Automated face recognition of rhesus macaques," J. Neurosci. Methods, vol. 300, pp. 157-165, Apr. 2018.

[57] I. M. Revina and W. R. S. Emmanuel, "A Survey on Human Face Expression Recognition Techniques," J. King Saud Univ. - Comput. Inf. Sci., vol. 33, no. 6, pp. 619-628, Jul. 2021.

[58] K. N. Kotsoglou and M. Oswald, "The long arm of the algorithm? Automated Facial Recognition as evidence and trigger for police intervention,” Forensic Sci. Int. Synerg., vol. 2, pp. 86-89, 2020.

[59] R. R. Bharadwaj, S. Belavadi, S. Gadicherla, V. A, S. Natarajan, and K. N. B. Murthy, "XNORNet and Minimum Barrier Detection for Efficient Face Recognition.," Procedia Comput. Sci., vol. 152, pp. 74-83, 2019.

[60] B. Hamdan and K. Mokhtar, "Face recognition using Angular Radial Transform,” J. King Saud Univ. - Comput. Inf. Sci., vol. 30, no. 2, pp. 141-151, Apr. 2018. 